\title{
AMNIOINFUSION: FROM TERMINATION OF PREGNANCY TO THERAPY
}

Amnioinfusion or infusion of saline into the amniotic cavity was first used as a technique for late termination of pregnancy in the 1960s (Jonas and Slate. Obstet Gynecol.1966;27:494-6). By the mid 1970s, transabdominal amnioinfusion of hypertonic $(20 \%)$ saline was the most widely used method of pregnancy termination after 14 weeks in the US. This technique was associated with around $10 \%$ morbidity including hemorrhage requiring transfusion, retained tissue requiring surgical removal, infection, coagulopathy, and hypernatremia (Ballard and Ballard. Am J Obstet Gynecol.1972;114:575-81). There were also risks of intravenous, intraperitoneal, or intramyometrial injection of the saline and thus amnioinfusion for abortion was abandoned in the 1980s in favour of prostaglandins (PGE2) which were more efficient and with fewer side effects.

Miyazaki and Taylor were the first to report on the use of saline amnioinfusion in 42 patients having repetitive variable or prolonged decelerations that did not respond to conventional therapy, such as maternal position changes and oxygen administration (Miyazaki and Taylor. Am J Obstet Gynecol.1983;146:670-8). The technique used normal saline or ringers lactate infused transcervically through a catheter into the uterine cavity, or transabdominally using a 'spinal' needle when membranes are intact. More recently, transcervical amnioinfusion was proposed for women presenting with thick meconium staining of the amniotic fluid to reduce the risk of perinatal death and meconium aspiration syndrome. Although the first results seemed encouraging (Hofmeyr et al.BJOG.1998;105:304-8), the 2006 UK National Institute for Health and Care Excellence (NICE) concluded that there was insufficient evidence to support the practice (www.nice.org.uk > guidance > ipg192) and it has rarely been used for this indication. More recent data from India have suggested that 
27 transcervical amnioinfusion in labour for meconium-stained amniotic fluid can be performed safely in a setup with limited neonatal care facilities, to decrease the incidence of caesarean deliveries and improve neonatal outcome (Choudhary and Bano. Arch Gynecol Obstet.2010;282:17-22).

Amnioinfusion was also used prophylactically in various conditions commonly associated with oligohydramnios to limit the risk of cord compression or oligohydramnios-related pulmonary hypoplasia (Figure) (Fisk et al. BJOG.1992;99:464-8) but was not found to improve neonatal outcomes. Two large trials have assessed whether infusion of fluid into the amniotic cavity could improve pregnancy outcome after second-trimester premature rupture of the membranes (AMIPROM trial Roberts et al.UOG 2014;43:490-9;PPROMEXIL-III trial van Teeffelen et al. BMC Pregnancy Childbirth. 2014; Apr 4;14:128). The first trial suggested an improvement in long-term healthy survival after amnioinfusion but neither of these studies showed a significant better outcome after amnioinfusion. In this issue, De Ruigh et al. (BJOG 2021) report on the long-term outcome of the PPROMEXIL-III trial.

42 Overall, of the 14 survivors (all born at a median gestational age of 24 weeks), $71 \%$ had no neurodevelopmental delay. The small sample size of this study does not allow to draw any definitive conclusions and survival without developmental delay nor respiratory problems did not differ between the treatment arm and controls with no amnioinfusion. From termination of pregnancy to therapy, amnioinfusion has still to 47 find its place in modern obstetrics. 
Word count: 497

50

51

52

BJOG since 1902 Perspectives on BJOG-19-0283R2

53

54

55

\section{Disclosure of interests}

56 The authors declare no conflicts of interest.

57

58

59

60 Eric Jauniaux, Professor, EGA Institute for Women's Health, Faculty of Population 61 Health Sciences, University College London, UK.

62

63 Both authors are BJOG scientific editors

64

67

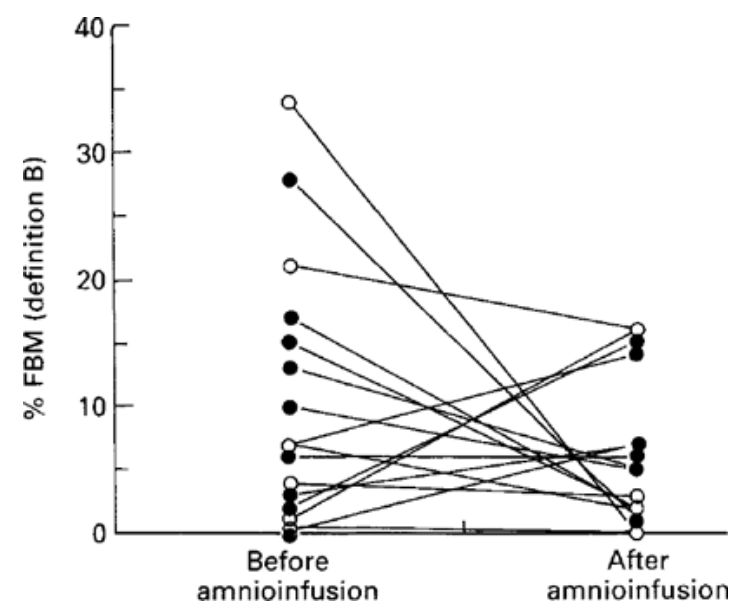

Figure: The incidence of FBM (=\% of time spent in FBM epochs) using definition B before and after amnioinfusion in 16 pregnancies. o pregnancies that leaked; • pregnancies that retained infused fluid (From Fisk et al., BJOG.1992;99:464-8). 